\section{Ankle arthroscopy in children}

PAJ de Leeuw, GMMJ Kerkhoffs

Academic Medical Centre, Amsterdam, Netherlands

doi: 10.1308/003588412X13171221500141

\section{CORRESPONDENCE TO}

Peter de Leeuw, E: p.a.deleeuw@amc.nl

\section{COMMENT ON}

Vasukutty NV, Akrawi H, Theruvil B, Uglow M. Ankle arthroscopy in

children. Ann R Coll Surg Engl 2011; 93: 232-235

doi 10.1308/003588411X564005

We read the article by Vasukutty et al with interest, care and appreciation, especially since reports in this field are rare. We have some additional questions for the authors.

1. One of the conclusions is the importance of a thorough physical examination. On that we agree. However, we feel that the approach described by the authors lacks structure. In the results section the authors report a lot of data but patient history, patient complaints and physical examination are mentioned interchangeably. In our opinion, it would have been useful to provide a simple diagram indicating history, complaints, physical examination, additional diagnostics, the examination under anaesthesia (EUA) and the arthroscopy as the golden standard, for each patient. In this way the combination of history, a good physical examination and additional diagnostics will allow for accurate diagnosis and neither EUA nor diagnostic arthroscopy of the ankle will prove necessary. ${ }^{1}$

We believe patient feedback on the physical examination is mandatory to differentiate between anterior ankle pain related to anterior ankle impingement and typical deep ankle pain related to a symptomatic osteochondral defect. $^{2}$ This also goes for the physical examination of a child.

2. We feel that the (long) term results for each patient remain unclear because the authors did not use validated outcome scores nor seemingly easy Likert or visual analogue scales that are developed for children. ${ }^{3}$ In order to be able to conclude that ankle arthroscopy 'is an effective way of treating post-traumatic ankle pathology', it would be wise to present intermediate and long-term results with validated outcome scores. Besides this, in $25 \%$ of the cases no traumatic event was reported.

3. We do not understand the thinking behind the setup of this research. Why did the authors not use magnetic resonance imaging (MRI) in every patient? Only then can the conclusion about the accuracy of MRI on diagnosing ankle impingement truly be made. What was the argument for using MRI? The authors do, however, perform an EUA in every patient - but for what purpose? We feel that the information from the patients' medical history and the findings on physical examination (eg recognisable or deep ankle pain, or even tenderness on palpation on the anteromedial distal tibial rim) is sufficient. There is therefore no need for EUA.

4. Complication rates in ankle arthroscopy vary widely but rates up to $17 \%$ have been reported. ${ }^{4}$ Several children underwent diagnostic arthroscopy, for which the authors refer to an article published in $1996 .^{5}$ Nowadays, ankle arthroscopy should be used only as a therapeutic tool since less invasive radiological modalities exist. ${ }^{1,6}$ How many children suffered from complications following the (diagnostic) ankle arthroscopy in their series? (See also arguments under point 1.)

\section{References}

1. Verhagen RA, Maas M, Dijkgraaf MG et al. Prospective study on diagnostic strategies in osteochondral lesions of the talus. Is MRI superior to helical CT? J Bone Joint Surg Br 2005; 87: 41-46.

2. van Dijk CN, van Bergen CJ. Advancements in ankle arthroscopy. J Am Acad Orthop Surg 2008; 16: 635-646.

3. Bieri D, Reeve RA, Champion GD et al. The Faces Pain Scale for the selfassessment of the severity of pain experienced by children: development, initial validation, and preliminary investigation for ratio scale properties. Pain 1990; 41: 139-150.

4. Barber FA, Click J, Britt BT. Complications of ankle arthroscopy. Foot Ankle 1990; 10: 263-266.

5. Stetson WB, Ferkel RD. Ankle arthroscopy: II. Indications and results. J Am Acad Orthop Surg 1996; 4: 24-34.

6. Stufkens SA, de Leeuw PA, van Dijk CN. Is there still a place for diagnostic ankle arthroscopy? European Musculoskeletal Review 2009; 4: 90-94.

\section{Authors' Response}

\section{Uglow}

University Hospital Southampton NHS Foundation Trust, UK doi: 10.1308/003588412X13171221500187

\section{CORRESPONDENCE TO}

Mike Uglow, E: mike.uglow@suht.swest.nhs.uk

I would like to thank de Leeuw and Kerkhoffs for their interest in our paper. In response to the questions raised I am pleased to provide the following information.

1. I agree entirely that an accurate examination complements the history. I also agree that no arthroscopy should be performed to make a diagnosis and no such procedures were performed in our series. An examination under anaesthesia (EUA) is performed immediately prior to an arthroscopy, the purpose of which is to see if the range of motion changes under anaesthesia and, more importantly, to confirm the stability of the ankle ligaments. Children are not necessarily able to allow a full ligament examination in the clinic environment and doing an EUA allows confident assessment of the structural stability of the joint.

2. We used the American Orthopaedic Foot and Ankle Society scoring method and believe that this is valid owing to the patients being adolescents and not young children. Pain assessment is an integral part of that scoring system and we feel that this was assessed. The patients were all assessed clinically and some of the follow-up 